Research Paper

\title{
Identification of the key genes and microRNAs in adult acute myeloid leukemia with FLT3 mutation by bioinformatics analysis
}

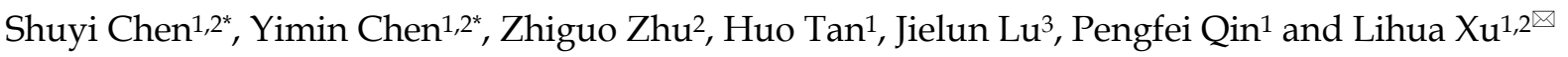 \\ 1. Department of Hematology, The First Affiliated Hospital of Guangzhou Medical University, Guangzhou, Guangdong 510000, China \\ 2. Department of Urology \& Minimally Invasive Surgery center, The First Affiliated Hospital of Guangzhou Medical University, Guangdong Key Laboratory \\ of Urology, Guangzhou Institute of Urology, Guangdong, China \\ 3. Department of Pediatrics, The First Affiliated Hospital of Guangzhou Medical University, Guangzhou, Guangdong 510000, China \\ ${ }^{*}$ Contributed equally to this work
}

$\square$ Corresponding author: Dr Lihua Xu, Department of Hematology, The First Affiliated Hospital of Guangzhou Medical University, 151 Yanjiang West Road, Yuexiu, Guangzhou, Guangdong 510000, China, E-mail: xlhua@gzhmu.edu.cn

(c) The author(s). This is an open access article distributed under the terms of the Creative Commons Attribution License (https://creativecommons.org/licenses/by/4.0/). See http:/ /ivyspring.com/terms for full terms and conditions.

Received: 2020.03.28; Accepted: 2020.05.03; Published: 2020.05.18

\begin{abstract}
Background: Associated with poor prognosis, FMS-like tyrosine kinase 3 (FLT3) mutation appeared frequently in acute myeloid leukemia (AML). Herein, we aimed to identify the key genes and miRNAs involved in adult AML with FLT3 mutation and find possible therapeutic targets for improving treatment.

Materials: Gene and miRNA expression data and survival profiles were obtained from The Cancer Genome Atlas (TCGA) and Gene Expression Omnibus (GEO) database. EdgeR of R platform was applied to identify the differentially expressed genes and miRNAs (DEGs, DE-miRNAs). Gene ontology (GO) and the Kyoto Encyclopedia of Genes and Genomes (KEGG) enrichment analyses were performed by Metascape and DAVID. And protein-protein interaction network, miRNA-mRNA regulatory network and clustering modules analyses were performed by STRING database and Cytoscape software.

Results: Survival analysis showed FLT3 mutation led to adverse outcome in AML. 24 DE-miRNAs (6 upregulated, 18 downregulated) and 250 DEGs (54 upregulated, 196 downregulated) were identified. Five miRNAs had prognostic value and the results matched their expression levels (miR-1-3p, miR-10a-3p, miR-10a-5p, miR-133a-3p and miR-99b-5p). GO analysis showed DEGs were enriched in skeletal system development, blood vessel development, cartilage development, tissue morphogenesis, cartilage morphogenesis, cell morphogenesis involved in differentiation, response to growth factor, cell-substrate adhesion and so on. The KEGG analysis showed DEGs were enriched in PI3K-Akt signaling pathway, ECM-receptor interaction and focal adhesion. Seven genes (LAMC1, COL3A1, APOB, COLIA2, APP, SPPI and FSTL1) were simultaneously identified by hub gene analysis and module analysis. SLC14A1, ARHGAP5 and PIK3CA, the target genes of miR-10a-3p, resulted in poor prognosis.

Conclusion: Our study successfully identified molecular markers, processes and pathways affected by FLT3 mutation in AML. Furthermore, miR-10a-3p, a novel oncogene, might involve in the development of FLT3 mutation adult AML by targeting SLC14A1, ARHGAP5 and PIK3CA.
\end{abstract}

Key words: bioinformatics analysis, acute myeloid leukemia, FLT3 mutation, differentially expressed miRNAs and genes, miR-10a-3p

\section{Introduction}

Acute myeloid leukemia (AML) is a common hematological malignancy which the incidence is increasing [1]. According to recent studies, a major reason why AML patients with low cure rate is drug resistance, usually manifested as relapse form remission [2]. Understanding the mutation status of various genes in early diagnosis could improve the effect of initial treatment. 
FMS-like tyrosine kinase 3 (FLT3), a type III receptor tyrosine kinase (RTK), is involved in multiple intracellular signaling pathways [3,4]. Activation of FLT3 plays an important role in hematopoietic cell survival, proliferation and differentiation $[5,6]$. However, FLT3 mutation is one of the most common genetic abnormalities in AML patients, accounting for about $30 \%-50 \%$. And the most common type of mutation in the FLT3 gene is an internal tandem duplication (FLT3/ITD) [7]. Associated with poor prognosis, mutated FLT3 is regarded as a promising therapeutic target for AML $[8,9]$. FLT3 inhibitors have approved for clinical use for mutant FLT3-positive AML in Japan and/or Europe and United states. But several resistance mechanisms of FLT3 inhibitors have been apparent in clinical studies [10]. Further studies are still required to find novel biomarkers for improving therapeutic strategy in AML with FLT3 mutation [11]. Chenglong Li et al. has investigated feature genes for predicting the FLT3/ITD mutation in pediatric and adult AML patients from the European Bioinformatics Institute (EBI) [12]. But the study was a lack of some analyses, such as protein-protein interaction network, predicted microRNAs (miRNAs) and miRNA-mRNA regulated network.

Herein, to provide novel targets for treatment, we identified the key genes and miRNAs and better understood the main biological processes associated with FLT3 mutations in adult AML by bioinformatics analysis.

\section{Materials and Methods}

\section{Data collection}

In order to compare genes and miRNAs expression between FLT3 mutation and wild-type adult AML patients, gene and miRNA expression profiles and corresponding survival profiles were obtained from The Cancer Genome Atlas (TCGA) database (https://gdc-portal.nci.nih.gov/) [13]. Simultaneously, the survival analysis data about FLT3 mutation and wild-type AML were provided by Bullinger $L$ et al [14]. The gene expression profile (GSE15434) for verification of target gene expression were downloaded from Gene Expression Omnibus (GEO) database (https://www.ncbi.nlm.nih .gov/geo/) [15]. The basic information of the downloaded dataset was performed in Table 1.

\section{Identification of differentially expressed miRNAs (DE-miRNAs) and differentially expressed genes (DEGs)}

The EdgeR was utilized to screen DE-miRNAs and DEGs according to user's guide $[16,17]$. $\mathrm{P}$ value $<0.05$ and $\mid \log _{2}$ fold change $(\mathrm{FC}) \mid \geq 1$ were set as the threshold values in DE-miRNAs, and $P$ value $<0.05$ and $\left|\log _{2} \mathrm{FC}\right| \geq 1.5$ were considered as cut-off criterion in DEGs.

\section{Enrichment analyses of DEGs}

Gene ontology (GO) term analysis for the DEGs was performed using Metascape (http:// metascape.org) [18], including biological process (BP), molecular function (MF) and cellular component (CC). KEGG pathways analysis for the DEGs was conducted by DAVID (https://david.ncifcrf.gov/ tools.jsp) [19]. $\mathrm{P}$ value $<0.05$ was considered statistically significant.

Table 1. Basic information of enrolled datasets

\begin{tabular}{|c|c|c|c|c|c|}
\hline Datasets & Type & $\begin{array}{l}\text { FLT3 } \\
\text { mutation } \\
\text { (n) }\end{array}$ & $\begin{array}{l}\text { FLT3 } \\
\text { wild-type } \\
\text { (n) }\end{array}$ & $\begin{array}{l}\text { Total } \\
\text { (n) }\end{array}$ & Purpose \\
\hline $\begin{array}{l}\text { Bullinger } \\
\text { Let al. }\end{array}$ & $\begin{array}{l}\text { survival } \\
\text { data }\end{array}$ & 70 & 106 & 176 & $\begin{array}{l}\text { Survival analysis of FLT3 } \\
\text { mutation AML }\end{array}$ \\
\hline \multirow[t]{3}{*}{ TCGA } & miRNA & 54 & 131 & 185 & DE-miRNAs \\
\hline & mRNA & 49 & 121 & 170 & DEGs \\
\hline & $\begin{array}{l}\text { survival } \\
\text { data }\end{array}$ & - & - & - & $\begin{array}{l}\text { Survival analysis of AML with } \\
\text { DE-miRNAs and target genes }\end{array}$ \\
\hline GSE15434 & mRNA & 90 & 161 & 251 & $\begin{array}{l}\text { Evaluation of target genes } \\
\text { expression }\end{array}$ \\
\hline
\end{tabular}

Abbreviations: TCGA: The Cancer Genome Atlas; FLT3: FMS-like tyrosine kinase 3, AML: acute myeloid leukemia; DE-miRNAs: differentially expressed miRNAs; DEGs: differentially expressed genes

\section{Protein-protein interaction (PPI) network, miRNA-mRNA regulatory network, hub genes and module analysis}

To analyze the connection among proteins, DEGs were uploaded to the Search Tool for the Retrieval of Interacting Genes (STRING, https://string-db.org/), which is a database that provides information on direct (physical) and indirect (functional) associations between different proteins [20]. This database is designed to provide important assessments and integration of functional partnerships and interactions that occur between proteins. The PPI network of DEGs and the regulatory network of miRNA-mRNA were visualized by Cytoscape software [21]. The cytoHubba plugin and Molecular Complex Detection (MCODE) plugin in Cytoscape were used to identify hub genes and screen modules of the PPI network [22, 23]. All parameters of the plugin were left at their default values. GO and KEGG enrichment of hub genes and genes in modules were also analyzed by Metascape.

\section{Evaluation of the prognostic value of DE-miRNAs and target genes of miR-10a-3p}

Survival curves were drawn by the Kaplan-Meier method. The hazard ratio (HR) with 95\% confidence intervals (CIs) and $\log$ rank $\mathrm{P}$ value 
were analyzed by the Cox proportional hazards regression model to compare the overall survival (OS) of AML with different miRNA or mRNA expression.

\section{Prediction of target genes of DE-miRNAs}

Target Scan [24], miRDB [25], miRPathDB [26] and miRWalk [27], target prediction databases, were both used to identify the target genes of DE-miRNAs. Moreover, the genes, predicted by the intersection of these four databases, were selected for further research.

\section{Statistical analysis}

All the statistical analyses were conduct with SPSS version 20.0 and GraphPad Prism version 8.0. P value $<0.05$ was indicated as statistical significant.

\section{Results}

\section{Procedure of bioinformatics analysis}

The flow chart of bioinformatics analysis was shown in Figure 1. Firstly, we identified DE-miRNAs and DEGs of AML with FLT3 mutations from TCGA database and evaluated the prognostic significance of DE-miRNAs to select valuable DE-miRNAs. Secondly, DEGs were conducted to perform GO and KEGG enrichment analyses. Then we constructed PPI network of DEGs and performed hub genes and module analysis. Moreover, the valuable DE-miRNAs and their predicted target genes were together to build the miRNA-mRNA regulatory network. Finally, miR-10a-3p and its target genes were chosen to further analyses.

\section{Identification of DE-miRNAs and DEGs}

First, the prognostic impact of FLT3 mutation in AML patients was evaluated using data from the article of Bullinger $\mathrm{L}$ et al. The survival analyses suggested that mutant FLT3 was significantly associated with poor overall survival (OS, P<0.001), relapse-free survival (RFS, $\mathrm{P}<0.01$ ), event-free survival (EFS, $\mathrm{P}<0.01)$ in AML patients (Figure 2). Therefore, it is important to identify crucial miRNA and mRNA and understand the relevant processes and pathways affected by FLT3 mutation.

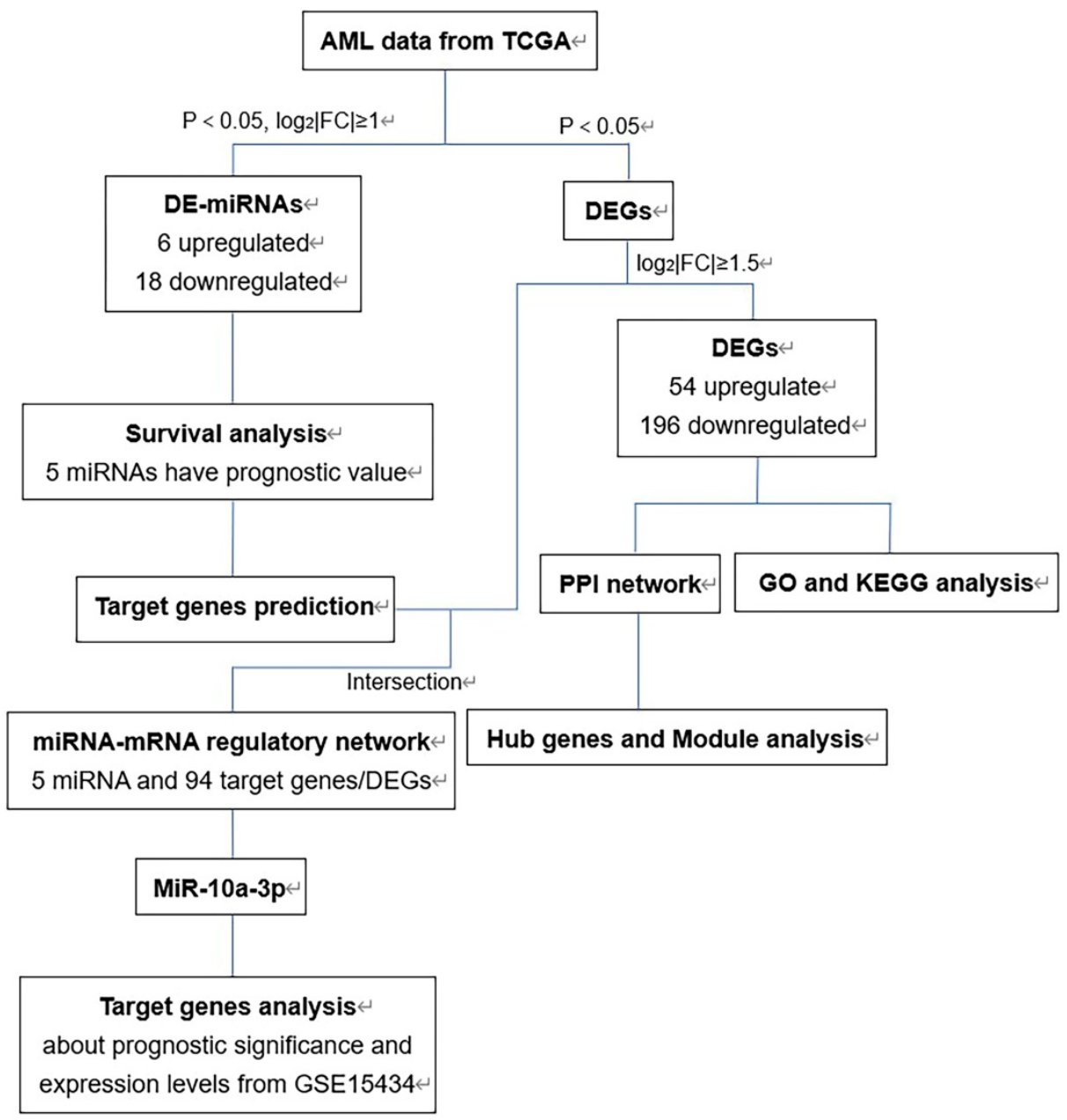

Figure 1. The flow chart of bioinformatics analysis. Abbreviations: AML, acute myeloid leukemia; TCGA, The Cancer Genome Atlas; DE-miRNAs, differentially expressed miRNAs; DEGs, differentially expressed genes; PPI: protein-protein interaction; GO: Gene ontology; KEGG: Kyoto Encyclopedia of Genes and Genomes. 
A

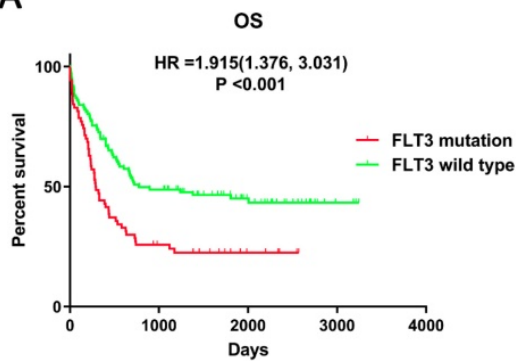

B

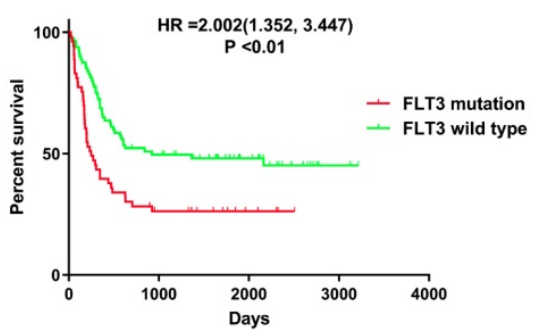

C

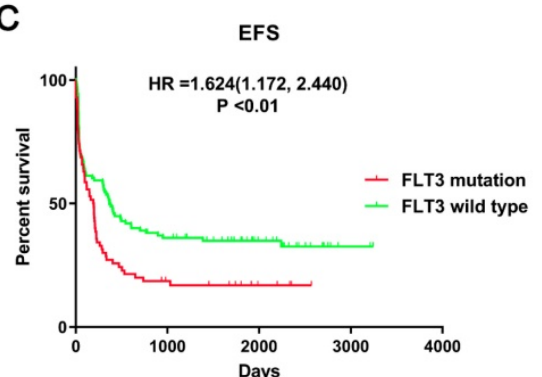

Figure 2. Survival analysis between FLT3 mutant and wild-type AML. Survival analysis was performed using data from Bullinger L et al. Abbreviations: OS: overall Survival; RFS: relapse-free survival; EFS: event-free survival.

Table 2. Top 20 GO terms enrichment analysis of DEGs by Metascape

\begin{tabular}{|c|c|c|c|c|c|c|c|}
\hline Gene & GO & Category & Description & Count & $\%$ & $\log 10(\mathrm{P})$ & $\log 10(q)$ \\
\hline$\overline{\downarrow \uparrow}$ & GO:0001501 & GO BP & skeletal system development & 36 & 14.63 & -19.06 & -14.71 \\
\hline$\downarrow$ & GO:0062023 & GO CC & collagen-containing extracellular matrix & 30 & 12.20 & -16.60 & -12.55 \\
\hline$\downarrow \uparrow$ & GO:0001568 & GO BP & blood vessel development & 34 & 13.82 & -12.31 & -8.82 \\
\hline$\downarrow \uparrow$ & GO:0051216 & GO BP & cartilage development & 19 & 7.72 & -12.27 & -8.82 \\
\hline$\downarrow$ & GO:0005833 & GO CC & hemoglobin complex & 7 & 3.63 & -11.85 & -8.34 \\
\hline$\downarrow$ & GO:0000904 & GO BP & cell morphogenesis involved in differentiation & 28 & 14.51 & -10.99 & -7.72 \\
\hline$\downarrow \uparrow$ & GO:0048729 & GO BP & tissue morphogenesis & 28 & 11.38 & -9.47 & -6.41 \\
\hline$\downarrow$ & GO:0097435 & GO BP & supramolecular fiber organization & 24 & 12.44 & -8.99 & -6.14 \\
\hline$\downarrow$ & GO:0019838 & GO MF & growth factor binding & 12 & 6.22 & -8.92 & -6.10 \\
\hline$\downarrow$ & GO:0044420 & GO CC & extracellular matrix component & 9 & 3.66 & -8.67 & -5.74 \\
\hline$\downarrow$ & GO:0005788 & GO CC & endoplasmic reticulum lumen & 18 & 7.32 & -8.48 & -5.60 \\
\hline$\downarrow$ & GO:0050808 & GO BP & synapse organization & 18 & 9.33 & -8.26 & -5.52 \\
\hline$\downarrow \uparrow$ & GO:0060536 & GO BP & cartilage morphogenesis & 7 & 2.85 & -7.87 & -5.16 \\
\hline$\downarrow$ & GO:0009611 & GO BP & response to wounding & 22 & 11.40 & -7.50 & -4.89 \\
\hline$\downarrow$ & GO:0001503 & GO BP & ossification & 19 & 7.72 & -7.50 & -4.85 \\
\hline$\downarrow$ & GO:0030003 & GO BP & cellular cation homeostasis & 21 & 10.88 & -7.16 & -4.58 \\
\hline$\downarrow$ & GO:0045177 & GO CC & apical part of cell & 16 & 8.29 & -7.02 & -4.46 \\
\hline$\downarrow$ & GO:0050839 & GO MF & cell adhesion molecule binding & 18 & 9.33 & -6.91 & -4.38 \\
\hline$\downarrow$ & GO:0070848 & GO BP & response to growth factor & 22 & 11.40 & -6.89 & -4.37 \\
\hline$\downarrow$ & GO:0031589 & GO BP & cell-substrate adhesion & 15 & 7.77 & -6.71 & -4.21 \\
\hline
\end{tabular}

Abbreviations: $\downarrow$ : downregulated genes; $\uparrow:$ upregulated genes; GO: gene ontology; BP: biological processes; CC: cellular components; MF: molecular function; DEGs: differentially expressed genes

Then we screened the DEGs and DE-miRNAs between FLT3 mutation and wild-type AML using sequencing data from TCGA database. A total of 24 DE-miRNAs (6 upregulated and 18 downregulated) and 250 DEGs (54 upregulated and 196 downregulated) were identified (Table S1). According to the results of overall survival analyses, 7 miRNA (miR-1-3p, miR-10a-3p, miR-10a-5p, miR-133a-3p, miR-99b-5p, miR-151a-5p and miR-598-3p) had prognostic significance in AML (Figure 3, Figure S1). However, the change on expression of miR-151a-5p and miR-598-3p (downregulated) did not match their adverse prognostic roles in OS analyses (miR-151a-5p: $\mathrm{HR}=1.847$, 95\%CI: 1.271-2.802, $\mathrm{P}=0.02$; miR-598-3p: $\mathrm{HR}=1.664$ ，95\%CI: 1.128-2.504， $\mathrm{P}=0.011)$. Therefore, miR-1-3p, miR-10a-3p, miR-10a-5p, miR-133a-3p and miR-99b-5p were selected as the candidate terms for further researches.

\section{GO and KEGG enrichment analysis of DEGs}

In order to further understand the pathway and process affected by identified DEGs, gene ontology
(GO) and Kyoto Encyclopedia of Gene and Genome (KEGG) analyses were respectively performed using Metascape and DAVID. The top 20 clusters with their representative enriched terms of GO analysis were shown in Table 2. The upregulated DEGs were significantly enriched in skeletal system development, blood vessel development, cartilage development, tissue morphogenesis and cartilage morphogenesis. While the downregulated DEGs were mainly enriched in skeletal system development, cell morphogenesis involved in differentiation, tissue morphogenesis, response to growth factor, cell-substrate adhesion and so on. These results were visualized by Metascape. A cluster heat map about enrichment analysis was shown in Figure 4A. Further, enrichment networks have been constructed to show the associations between GO terms. In the networks, the nodes were represented as different terms, which were colored by their cluster ID (Figure 4B), P value (Figure $4 \mathrm{C}$ ) and the identities of the gene lists (Figure 4D). 
A

miR-1-3p

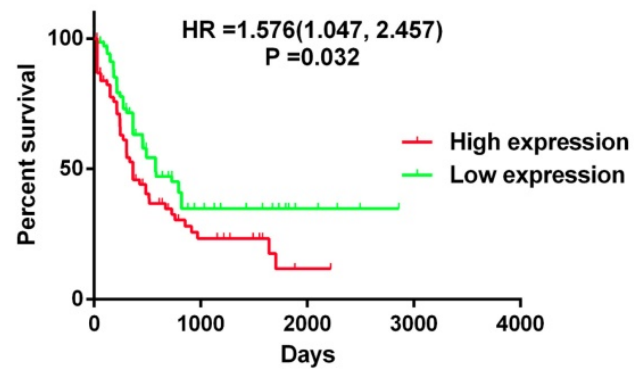

C

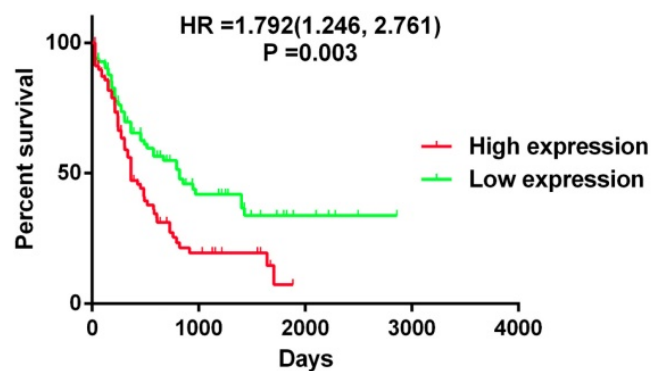

E

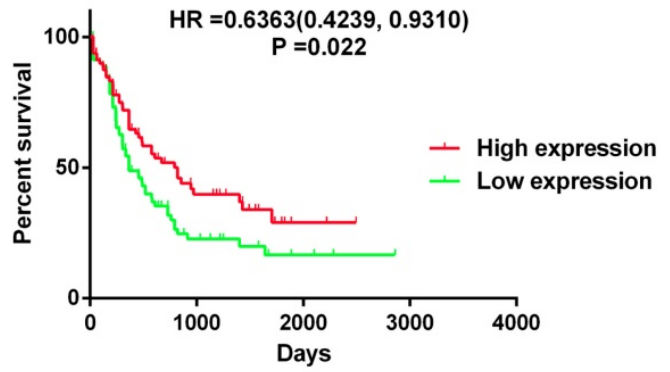

miR-598-3p

G

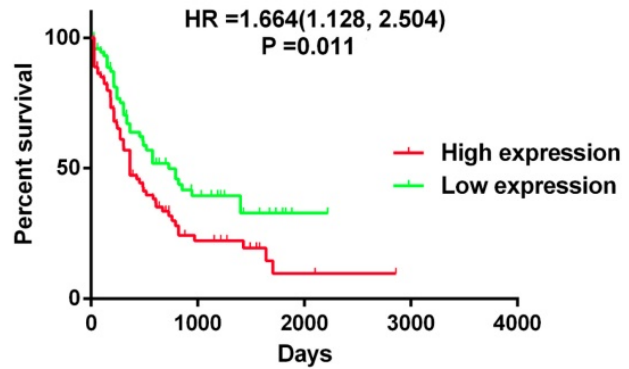

B

miR-10a-3p

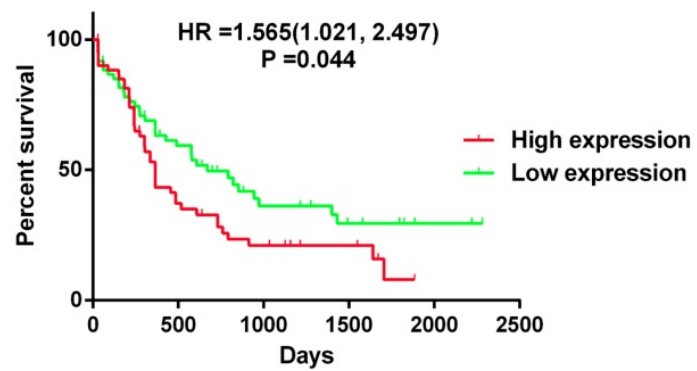

miR-133a-3p

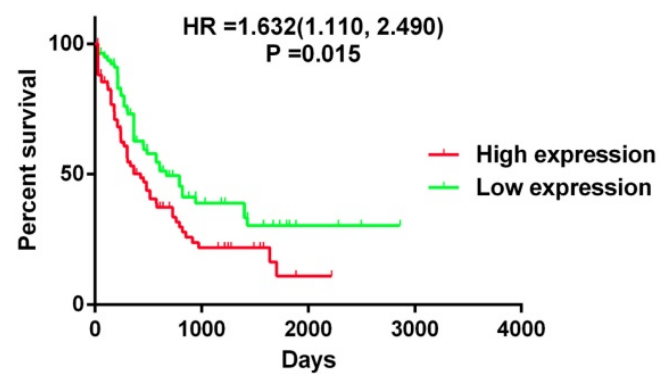

F

miR-151a-5p

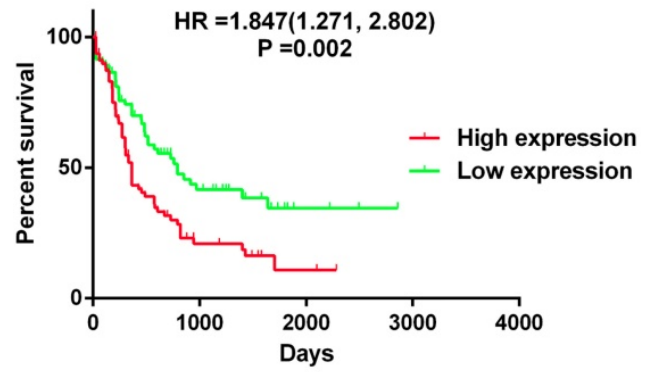

Figure 3. Prognostic significance of seven DE-miRNAs. Kaplan-Meier survival curves of overall survival (OS) in AML was performed using TCGA dataset. The top 50\% miRNA expression was defined as high expression group and the rest was defined as low expression group. The hazard ratio (HR) with $95 \%$ confidence intervals (Cls) were analyzed by the Cox proportional hazards regression model. Abbreviations: DE-miRNAs: differentially expressed miRNAs; HR: hazard ratio.

KEGG pathway analysis was conducted for total DEGs by DAVID. The result showed DEGs were obviously enriched in PI3K-Akt signaling pathway, ECM-receptor interaction, focal adhesion Protein digestion and absorption, Chemokine signaling pathway and so on (Figure 5, Table 3).

\section{PPI network and module analysis of DEGs}

To evaluate the association of DEGs, protein-protein interactome (PPI) network were performed using STRING and Cytoscape software (Figure 6A). The top 20 hub genes were detected by cytoHubba plugin used 12 different algorithms (Table 
S2). Then, detected by more than five algorithms, 15 hub genes with higher degree of connectivity were selected to build the hub gene PPI network (Figure $6 \mathrm{~B})$. The enrichment analysis showed that the cancer-related processes hub genes enrich in were extracellular structure organization, leukocyte migration, cell-substrate adhesion, negative regulation of cell migration, regulation of cell adhesion and so on.
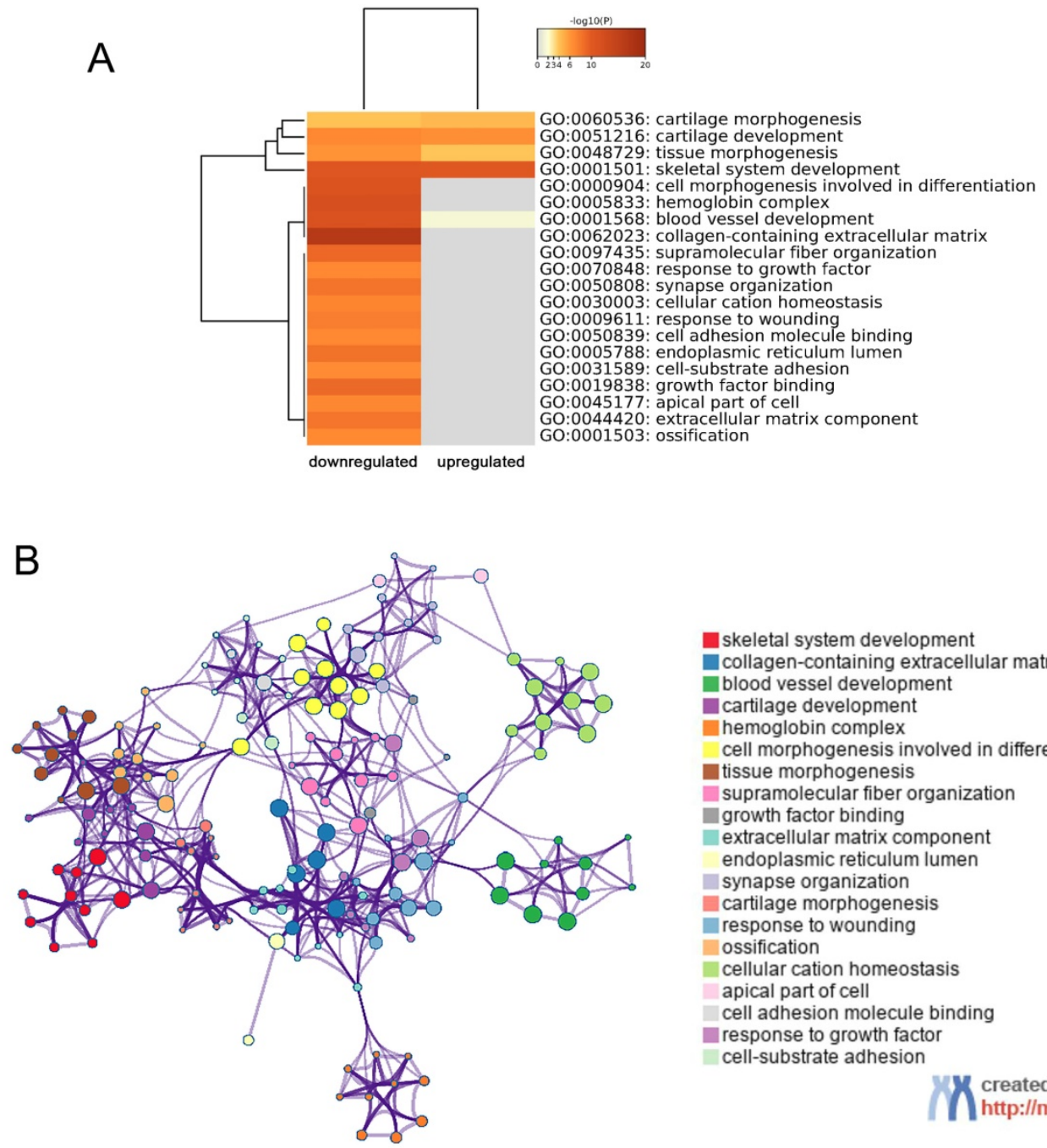

skeletal system development

collagen-containing extracellular matrix

blood vessel development

cartilage development

hemoglobin complex

cell morphogenesis involved in differentiation

tissue morphogenesis

supramolecular fiber organization

growth factor binding

extracellular matrix component

endoplasmic reticulum lumen

synapse organization

cartilage morphogenesis

response to wounding

ossification

cellular cation homeostasis

apical part of cell

cell adhesion molecule binding

response to growth factor

cell-substrate adhesion

C

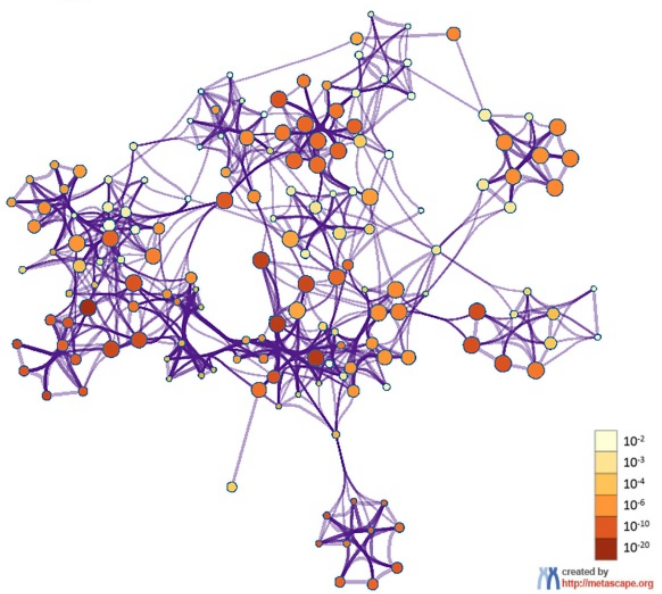

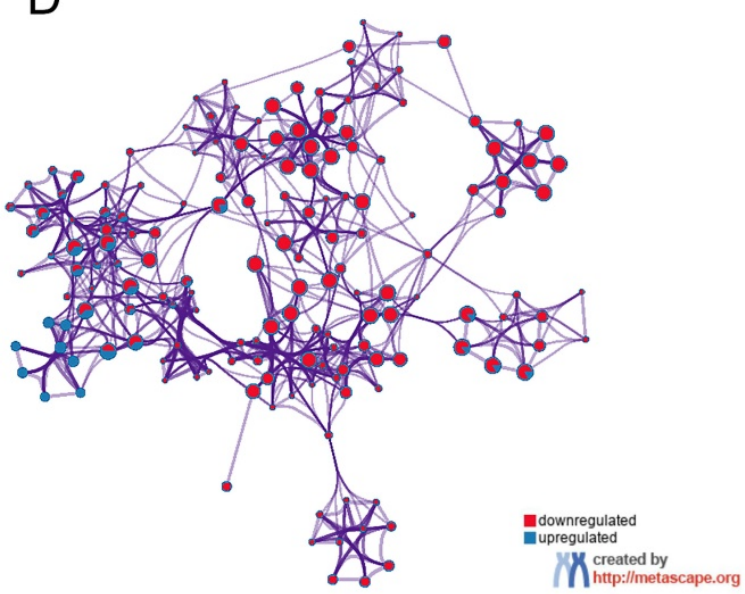

Figure 4. GO enrichment and interactome analysis of DEGs. A, Heatmap of enriched terms for downregulated and upregulated DEGs colored by $P$ values was visualized by Metascape. B-D, Network of enriched terms: (B) colored by enriched terms, (C) colored by P value, (D) color-coded based on the identities of the gene lists. Abbreviations: DEGs: differentially expressed genes; GO: gene ontology. 


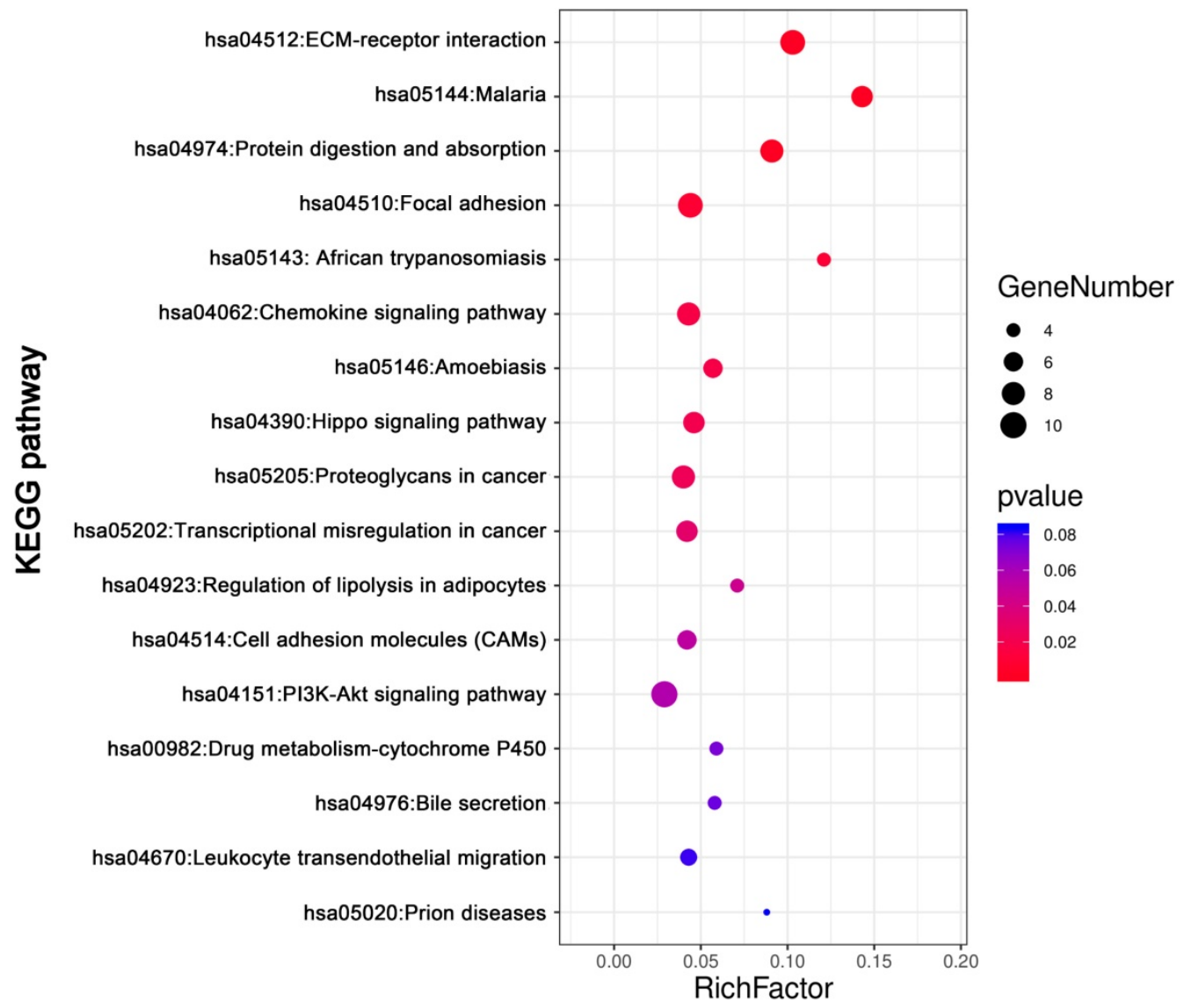

Figure 5. The KEGG pathways analysis of DEGs. The KEGG pathways enrichment analysis of differentially expressed genes was perform by DAVID. Abbreviations: KEGG: Kyoto Encyclopedia of Genes and Genomes.

Table 3. The KEGG pathways enrichment analysis of DEGs by DAVID

\begin{tabular}{|c|c|c|c|c|c|}
\hline ID & Term & Count & $\%$ & P value & Genes \\
\hline hsa04151 & PI3K-Akt signaling pathway & 10 & 0.030149 & 0.056461 & PTK2, COL3A1, COL6A3, COL1A2, COL6A2, GNG11, COL1A1, LAMC1, SPP1, COL4A5 \\
\hline hsa04512 & ECM-receptor interaction & 9 & 0.027134 & 2.95E-05 & SDC1, COL3A1, COL6A3, COL1A2, COL6A2, COL1A1, LAMC1, SPP1, COL4A5 \\
\hline hsa04510 & Focal adhesion & 9 & 0.027134 & 0.008848 & PTK2, COL3A1, COL6A3, COL1A2, COL6A2, COL1A1, LAMC1, SPP1, COL4A5 \\
\hline hsa04974 & Protein digestion and absorption & 8 & 0.024119 & 2.38E-04 & COL14A1, ATP1B2, COL3A1, COL6A3, COL1A2, COL6A2, COL1A1, COL4A5 \\
\hline hsa04062 & Chemokine signaling pathway & 8 & 0.024119 & 0.01651 & CCL1, PTK2, PARD3, CCL14, ADCY2, TIAM1, GNG11, CXCL12 \\
\hline hsa05205 & Proteoglycans in cancer & 8 & 0.024119 & 0.023575 & WNT7B, PTK2, CTTN, SDC1, TIAM1, IGF2, DCN, TIMP3 \\
\hline hsa05144 & Malaria & 7 & 0.021104 & 6.11E-05 & GYPB, VCAM1, SDC1, GYPA, HBA2, HBA1, HBB \\
\hline hsa04390 & Hippo signaling pathway & 7 & 0.021104 & 0.020389 & WNT7B, PARD3, FRMD6, CTGF, DLG3, TEAD1, GLI2 \\
\hline hsa05202 & Transcriptional misregulation in cancer & 7 & 0.021104 & 0.031476 & PROM1, EYA1, PTK2, TSPAN7, PBX1, HMGA2, MLLT3 \\
\hline hsa05146 & Amoebiasis & 6 & 0.018089 & 0.017315 & PTK2, COL3A1, COL1A2, COL1A1, LAMC1, COL4A5 \\
\hline hsa04514 & Cell adhesion molecules (CAMs) & 6 & 0.018089 & 0.05155 & VCAM1, SDC1, PTPRM, CD34, NLGN4X, CDH5 \\
\hline hsa04670 & Leukocyte transendothelial migration & 5 & 0.015074 & 0.08114 & VCAM1, PTK2, CXCL12, CDH5, THY1 \\
\hline hsa05143 & African trypanosomiasis & 4 & 0.012059 & 0.011226 & VCAM1, HBA2, HBA1, HBB \\
\hline hsa04923 & Regulation of lipolysis in adipocytes & 4 & 0.012059 & 0.045163 & ADCY2, ADRB1, FABP4, PRKG2 \\
\hline hsa00982 & Drug metabolism - cytochrome P450 & 4 & 0.012059 & 0.072398 & FMO2, FMO3, UGT2B11, GSTT1 \\
\hline hsa04976 & Bile secretion & 4 & 0.012059 & 0.074937 & ADCY2, ATP1B2, SLC4A4, ABCG2 \\
\hline hsa05020 & Prion diseases & 3 & 0.009045 & 0.083993 & C7, C1QB, LAMC1 \\
\hline
\end{tabular}

Abbreviations: KEGG: Kyoto Encyclopedia of Genes and Genomes; DEGs: differentially expressed genes.

In addition, 9 modules in PPI network were detected by the MCODE plugin. We screened the top 3 significant modules to further analyses. The enrichment analysis demonstrated that genes in modules were mainly enriched in hemoglobin binding, extracellular structure organization, platelet degranulation, ECM-receptor interaction, tissue morphogenesis, and definitive hemopoiesis (Figure 6
C). Seven genes (LAMC1, COL3A1, APOB, COL1A2, APP, SPP1 and FSTL1) were simultaneously identified by hub gene analysis and module analysis.

\section{miRNA-mRNA regulatory network analysis}

Four target prediction databases were utilized to identify the target genes of five candidate DE-miRNAs (Figure 7A). 
B
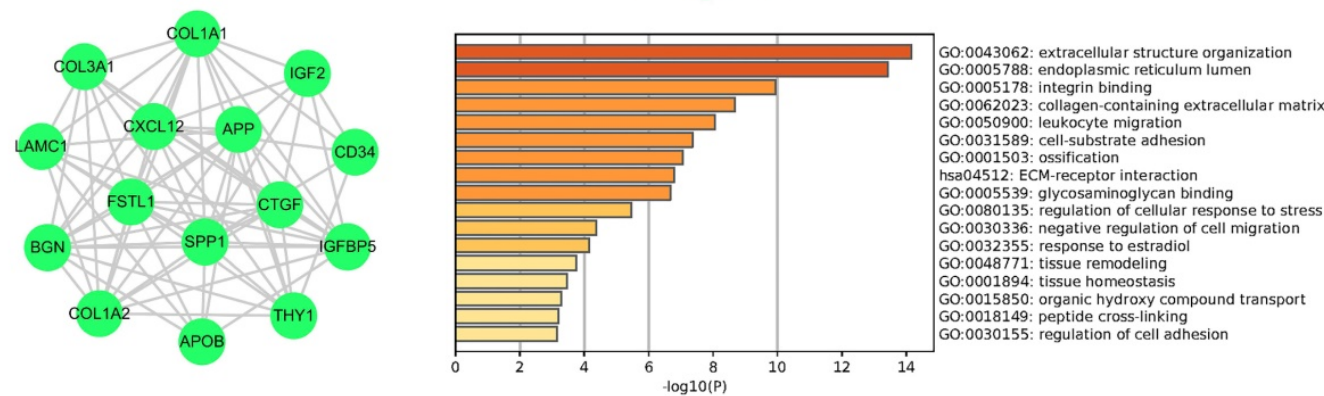

C
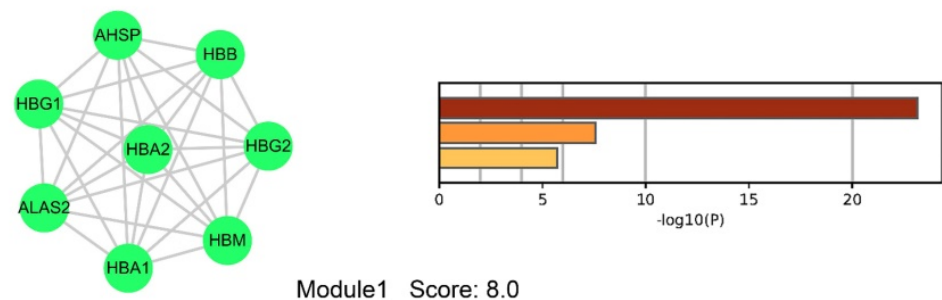

G0:0005833: hemoglobin complex GO:0071682: endocytic vesicle lumen GO:0030492: hemoglobin binding

Module1 Score: 8.0
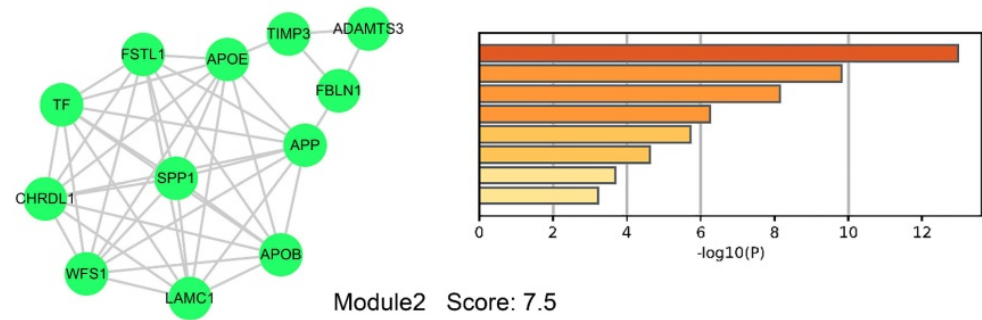

GO:0005788: endoplasmic reticulum lumen GO:0043062: extracellular structure organization GO:0008201: heparin binding

GO:0062023: collagen-containing extracellular matrix GO:0034363: intermediate-density lipoprotein particle GO:0002576: platelet degranulation hsa04512: ECM-receptor interaction

Module2 Score: 7.5
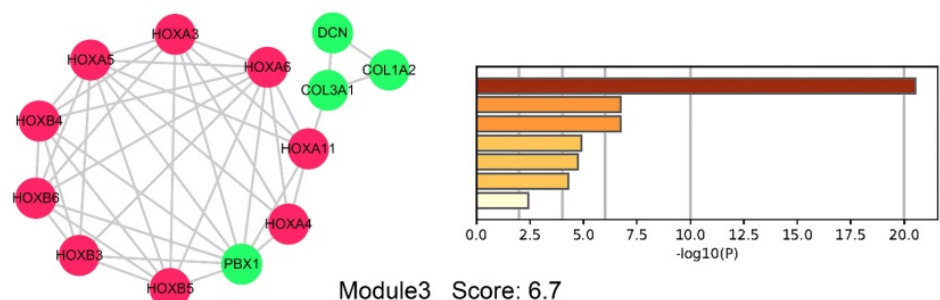

GO:0048706: embryonic skeletal system development

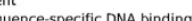
GO:0030878: thyroid gland development GO:0005581: collagen trime

GO:0048729: tissue morphogenesis

GO:0060216: definitive hemopoiesis GO:0016525: negative regulation of angiogenesis

Module3 Score: 6.7

Figure 6. PPI network, hub genes network and modules analyses of DEGs. A, PPI network of DEGs. B, The network of 15 hub genes with a higher degree of connectivity and enrichment analysis of these genes. C, Genes of top 3 modules were performed GO and KEGG enrichment analysis by Metascape. Nodes were color-coded based on the expression of DEGs (red, upregulated; green, downregulated).Abbreviations: DEGs: differentially expressed genes; GO: Gene ontology; KEGG: Kyoto Encyclopedia of Genes and Genomes, PPI: protein-protein interaction. 
A

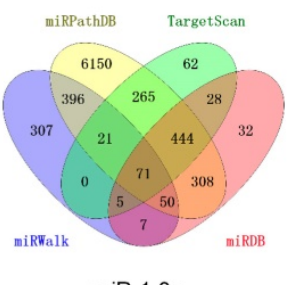

miR-1-3p

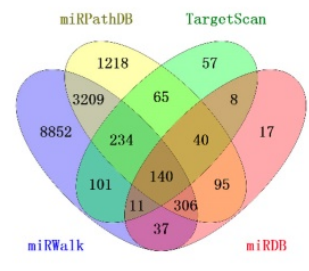

miR-133a-3p

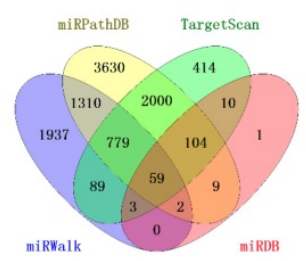

miR-10-3p

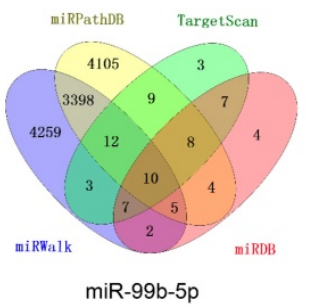

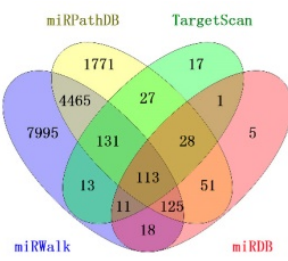

$\operatorname{miR}-10-5 p$

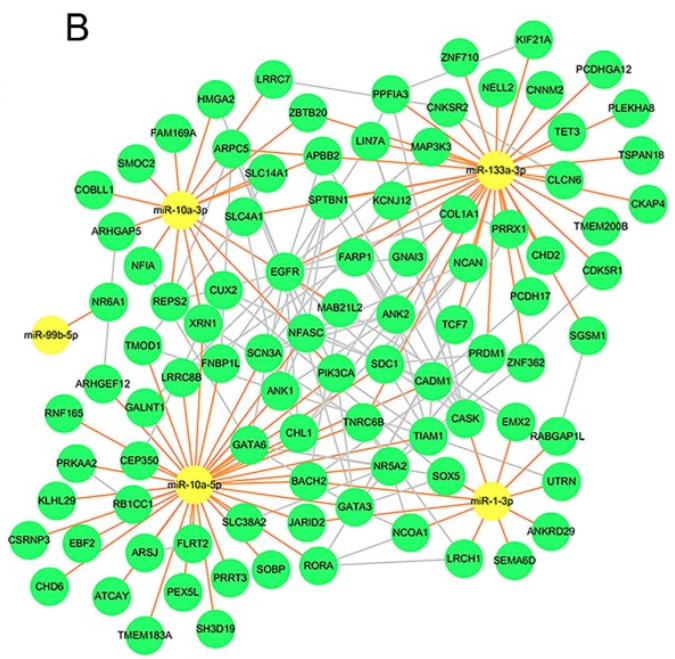

Figure 7. miRNA-mRNA regulatory network. A, Diagrams illustrating in prediction of target genes of five candidate miRNAs. B, miRNA-mRNA regulatory network. Nodes were color-coded based on components (yellow, DE-miRNAs; green, target DEGs). The orange lines indicated the regulation relationship between DE-miRNAs and their targets DGEs. The silver lines indicated the relationship between DEGs. Abbreviations: DE-miRNAs: differentially expressed miRNAs: DEGs, differentially expressed genes.

The total target genes were matched with DGEs $(\mathrm{P}<0.05$, not limited fold change), while the target genes of upregulated miRNA were corresponded with downregulated genes and target gens of downregulated miRNA were corresponded with upregulated genes. Herein, we totally identified 393 target genes and there were 94 genes expressing differently between FLT3 mutation and wild-type AML. Then, miRNA-mRNA regulatory network was constructed with DE-miRNA $(\mathrm{n}=5)$ and target gens $(n=94)$. It showed that miR-1-3p, miR-10a-3p, miR-10a-5p and miR-133a-3p had rich external connections (Figure 7B). Finally, three transcription factors, RORA, CUX2 and ZNF362, were found in DE-miRNAs-DEGs regulatory network using by OmicsBean (http:/ / www.omicsbean.cn).

\section{Target genes analysis of miR-10a-3p}

Some studies have widely investigated the identified key miRNA in cancers except miR-10a-3p. For example, miR-1-3p acted as a tumor suppressor in acute myeloid leukemia [28], bladder cancer [29], non-small-cell lung cancer [30], hepatocellular carcinoma [31] and so on. Zhi Y et al. reported that high miR-10a-5p expression was associated with poorer overall survival of AML patients [32]. miR-133a-3p played a regulated role in prostate cancer [33], ovarian cancer [34] and breast cancer [35]. And miR-99b-5p expression was related with gastric cancer [36], colorectal cancer [37] and clear cell renal cell carcinoma [38]. However, the molecular mechanism of miR-10a-3p in cancer was still unclear. So, we selected miR-10a-3p for further study.

In the miRNA-mRNA regulatory network, miR-10a-3p had 15 target genes: HMGA2, NFIA, CUX2, SLC14A1, LRRC7, APBB2, SMOC2, FAM169A,
ARHGAP5, COBLL1, MAB21L2, ZBTB20, REPS2, XRN1 and PIK3CA, also acted as downregulated genes in FLT3 mutation AML of TCGA. Then these target genes and miR-10a-3p were performed correlation analysis. The results shown that miR-10a-3p had negative correlations with HMGA2, NFIA, CUX2, SLC14A1, APBB2, ARHGAP5, COBLL1, REPS2, XRN1 and PIK3CA (P<0.05, Figure 8, Figure S2), of which SLC14A1, ARHGAP5, PIK3CA had prognostic significance in OS of AML patients $(\mathrm{P}<0.05$, Figure 8, Figure S2). Simultaneously, expression of these three target genes was verified in GSE15434. Expression of SLC14A1 $(\mathrm{P}<0.001)$ and ARHGAP5 $(\mathrm{P}=0.033)$ were both reduced in AML with FLT3 mutation, but there was no obvious difference of PIK3CA ( $\mathrm{P}=0.647$ ) expression (Figure 8).

\section{Discussion}

Acute myeloid leukemia (AML) is a common hematological malignancy which the incidence is increasing [1]. As a highly heterogeneous disease, AML is associated with many different cytogenetic abnormalities and genetic alterations detected at diagnosis. Hence, the study of valuable biomarkers is beneficial to enhance our understanding of AML pathogenesis. FMS-like tyrosine kinase 3 (FLT3) mutation is one of the most common genetic abnormalities in AML patients [7]. Survival analyses indicated that FLT3 mutation was significantly associated with poor OS, RFS and EFS using data provided by Bullinger L et al. [14], which was consisted with previous studies [39, 40]. Therefore, further investigation is important for better understanding the biological roles of FLT3 mutations in AML. 

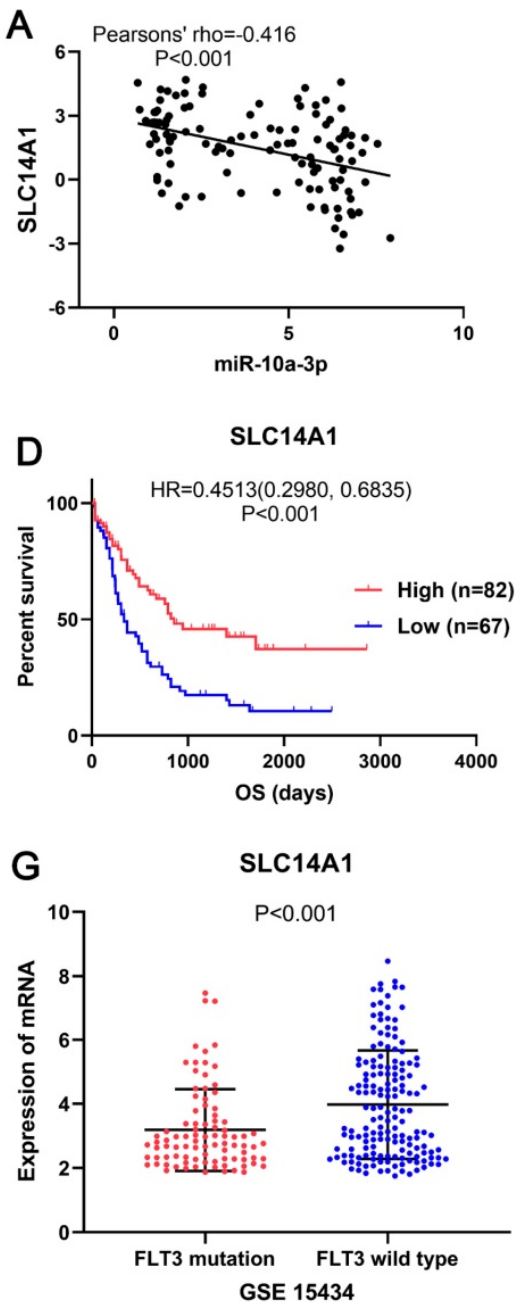

B

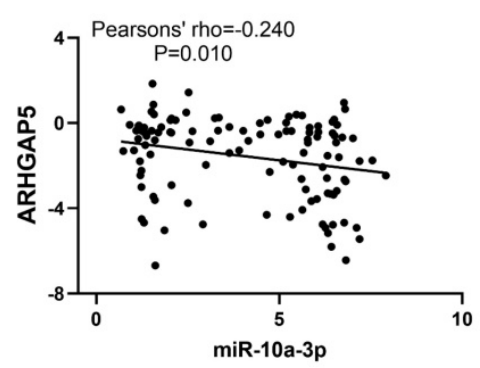

E

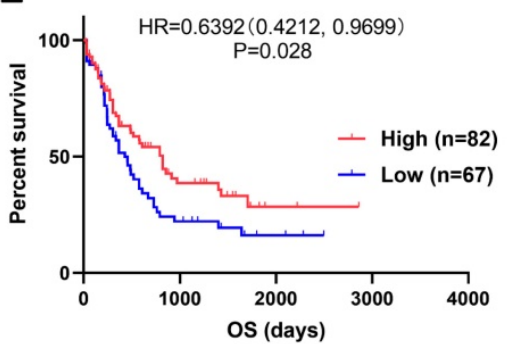

$\mathrm{H}$

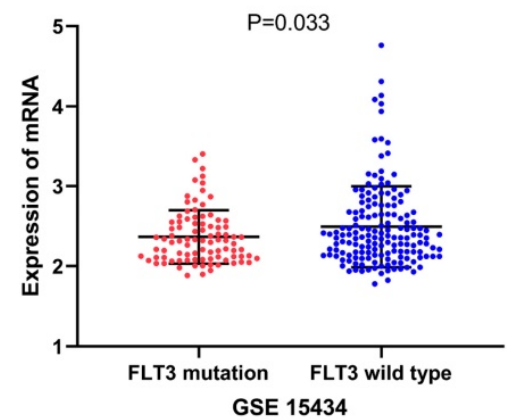

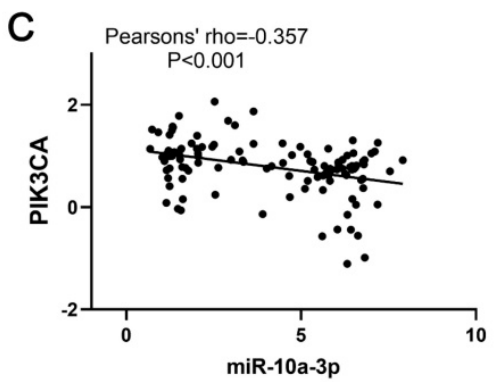
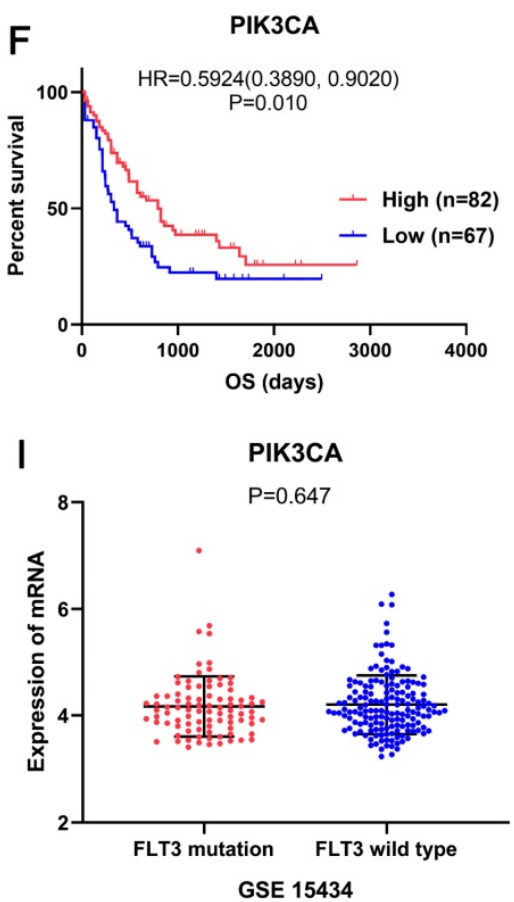

Figure 8. SLC14A1, ARHGAP5 and PIK3CA may be the target genes of miR-10a-3p involved in AML with FLT3 mutation. The correlation analyses showed miR-10a-3p had significant negative correlations with SLC14A1 (pearsons' rho=-0.42), ARHGAP5 (pearsons' rho=-0.24) and PIK3CA (pearsons' rho=-0.36) in AML with FLT3 mutation from TCGA. Survival analyses shown that SLC14A1, ARHGAP5 and PIK3CA had better overall survival (OS) of AML patients form TCGA and had different mRNA expression levels between FLT3 mutation and wild-type AML from GSE15434. Abbreviations: TCGA: The Cancer Genome Atlas; OS: overall survival.

In present study, we firstly used mRNA and miRNA sequencing data from TCGA database to analyze the molecular correlates of FLT3 mutations in adult AML. A total of 24 DE-miRNAs (6 up-regulated and 18 down-regulated) and 250 DEGs (54 up-regulated and 196 down-regulated) were identified using bioinformatics analysis. To understand the changes in processes and pathways FLT3 mutation caused, GO terms and KEGG pathway enrichment analyses were performed. The results of GO analyses showed DEGs were notably abundant in skeletal system development, blood vessel development, cartilage development, tissue morphogenesis, cartilage morphogenesis, cell morphogenesis involved in differentiation, response to growth factor, cell-substrate adhesion and so on. It is suggested that FLT3 mutation affected leukemia via these processes.

The KEGG pathway analysis showed that DEGs were obviously enriched in PI3K-Akt signaling pathway, ECM-receptor interaction and focal adhesion. Previous studies had reported that PI3K-Akt signaling pathway was involved in AML $[41,42]$. But the roles of ECM-receptor interaction pathway and focal adhesion pathway in AML were still unclear. Kim $\mathrm{SH}$ et al demonstrated that ECM-receptor interaction led to direct or indirect control of cell adhesion, migration, differentiation, proliferation and apoptosis [43]. ECM-receptor interaction was reported to be related to esophageal squamous cell carcinoma [44] and epithelial ovarian cancer [45]. In addition, focal adhesion signaling hubs critically regulated cell behavior, impacted on tumor cell survival and served as potential cancer targets [46].

PPI network showed DEGs involved in FLT3 mutation were rich interactions. The top hub genes detected by more than five algorithms included LAMC1, COL3A1, COL1A1, IGF2, CD34, IGFBP5, THY1, APOB, COL1A2, BGN, CXCL12, APP, CTGF, 
SPP1 and FSTL1. By enrichment analysis, these hub genes were associated with extracellular structure organization, leukocyte migration, cell-substrate adhesion, negative regulation of cell migration, regulation of cell adhesion and so on. In addition, the analyses demonstrated that genes in the top 3 significant modules were mainly enriched in hemoglobin binding, extracellular structure organization, platelet degranulation, ECM-receptor interaction, tissue morphogenesis, and definitive hemopoiesis. Seven genes (LAMC1, COL3A1, APOB, COL1A2, APP, SPP1 and FSTL1) were simultaneously identified by hub gene analysis and module analysis. Therefore, these genes might be crucial in FLT3 mutation AML.

We identified 24 DE-miRNAs and 5 of them had prognostic value and the results matched their expression levels. Four miRNAs (miR-1-3p, miR-10a-3p, miR-10a-5p and miR-133a-3p) resulted in adverse outcome and miR-99b-5p played positive prognosis in AML. Four target prediction databases were used to identify the targets of DE-miRNAs. Then, miRNA-mRNA regulatory network revealed that miR-1-3p, miR-10a-3p, miR-10a-5p and miR-133a-3p had rich external connections, while miR-99b-5p have few external connections. miR-1-3p acted as a tumor suppressor in acute myeloid leukemia [28], bladder cancer [29], non-small-cell lung cancer [30], hepatocellular carcinoma [31] and so on. Zhi $Y$ et al. reported that high miR-10a-5p expression was associated with poorer overall survival of AML patients [32]. miR-133a-3p played a regulated role in prostate cancer [33], ovarian cancer [34] and breast cancer [35]. And miR-99b-5p expression was related with gastric cancer [36], colorectal cancer [37] and clear cell renal cell carcinoma [38]. However, the study about role of miR-10a-3p in cancer was still lacking. So, we selected miR-10a-3p for further study.

Among the target genes of miR-10a-3p, SLC14A1, ARHGAP5, PIK3CA had significant negative correlation with it and led to better overall survival in AML. Simultaneously, expression of these three target genes was verified in GSE15434. Expression of SLC14A1 and ARHGAP5 were both reduced in AML with FLT3 mutation, but there was no obvious difference of PIK3CA expression. Human solute carrier family 14 member 1 (SLC14A1) gene was crucial to the kidney's ability to concentrate urine and also expressed on red blood cells [47]. Studies has reported that SLC14A1 was regard as a novel target for human urothelial cancer [48] and urinary bladder cancer [49]. However, Rho GTPase activating protein 5 (ARHGAP5) was identified as an oncogene that affected cell migration and invasion in cervical cancer [50], gastric cancer [51] and nasopharyngeal carcinoma [52]. Phosphatidylinositol 3-kinase catalytic subunit alpha (PIK3CA) mutation has found in hematological malignancies [53], including acute myeloid leukemia [54] and diffuse large B cell lymphoma [55]. In addition, studies of SLC14A1 and ARHGAP5 related to AML had not been reported. According to the results, the expression levels of SLC14A1 and ARHGAP5 were downregulated in FLT3 mutation AML patients compared with the wild-type, which were required to be confirm by further experiment.

\section{Conclusion}

Our study indicated that mutant FLT3 result in poor prognosis in adult AML which was in line with previous reports. We successfully identified molecular markers, processes and pathways affected by FLT3 mutation in AML. Furthermore, miR-10a-3p, a novel biomarker, might involve in the development of FLT3 mutation AML by targeting SLC14A1, ARHGAP5 and PIK3CA. However, further experiments are still required to support our results.

\section{Supplementary Material}

Supplementary figures and tables. http://www.medsci.org/v17p1269s1.pdf

\section{Acknowledgments}

The authors thank The Cancer Genome Atlas (TCGA) and Gene Expression Omnibus (GEO) database for their sharing of the AML sequencing dataset and corresponding survival profiles.

\section{Funding}

This study was supported by grants from National Natural Science Foundation of China (81672661, 81870113), Guangzhou medical and health technology program (20181A011062) and Guangdong Province Natural Science Foundation (2017A03031 3858).

\section{Data Availability}

The data used to support findings of this study are available from the corresponding author upon request.

\section{Competing Interests}

The authors have declared that no competing interest exists.

\section{References}

1. Siegel RL, Miller KD, Jemal A. Cancer statistics, 2020. CA Cancer J Clin. 2020; 70: 7-30

2. Estey EH. Acute myeloid leukemia: 2019 update on risk-stratification and management. Am J Hematol. 2018; 93: 1267-91.

3. Schlessinger J. Cell signaling by receptor tyrosine kinases. Cell. 2000; 103: 211-25. 
4. Rosnet $\mathrm{O}$, Mattei MG, Marchetto S, Birnbaum D. Isolation and chromosomal localization of a novel FMS-like tyrosine kinase gene. Genomics. 1991; 9: 380-5.

5. Rosnet O, Schiff C, Pebusque MJ, Marchetto S, Tonnelle C, Toiron Y, et al. Human FLT3/FLK2 gene: cDNA cloning and expression in hematopoietic cells. Blood. 1993; 82: 1110-9.

6. Lyman SD, James L, Vanden Bos T, de Vries P, Brasel K, Gliniak B, et al. Molecular cloning of a ligand for the flt3/flk-2 tyrosine kinase receptor: a proliferative factor for primitive hematopoietic cells. Cell. 1993; 75: 1157-67.

7. Gilliland DG, Griffin JD. The roles of FLT3 in hematopoiesis and leukemia. Blood. 2002; 100: 1532-42.

8. Dohner H, Estey E, Grimwade D, Amadori S, Appelbaum FR, Buchner T, et al. Diagnosis and management of AML in adults: 2017 ELN recommendations from an international expert panel. Blood. 2017; 129: 424-47.

9. Kiyoi H. Flt3 Inhibitors: Recent Advances and Problems for Clinical Application. Nagoya J Med Sci. 2015; 77: 7-17.

10. Weisberg E, Barrett R, Liu Q, Stone R, Gray N, Griffin JD. FLT3 inhibition and mechanisms of drug resistance in mutant FLT3-positive AML. Drug Resist Updat. 2009; 12: 81-9.

11. Kiyoi H, Kawashima N, Ishikawa Y. FLT3 mutations in acute myeloid leukemia: Therapeutic paradigm beyond inhibitor development. Cancer Sci. 2020; 111: 312-22.

12. $\mathrm{Li} \mathrm{C}$, Zhu B, Chen J, Huang X. Feature genes predicting the FLT3/ITD mutation in acute myeloid leukemia. Mol Med Rep. 2016; 14: 89-94.

13. Cancer Genome Atlas Research N, Ley TJ, Miller C, Ding L, Raphael BJ, Mungall AJ, et al. Genomic and epigenomic landscapes of adult de novo acute myeloid leukemia. N Engl J Med. 2013; 368: 2059-74.

14. Bullinger L, Ehrich M, Dohner K, Schlenk RF, Dohner H, Nelson MR, et al. Quantitative DNA methylation predicts survival in adult acute myeloid leukemia. Blood. 2010; 115: 636-42.

15. Klein HU, Ruckert C, Kohlmann A, Bullinger L, Thiede C, Haferlach T, et al. Quantitative comparison of microarray experiments with published leukemia related gene expression signatures. BMC Bioinformatics. 2009; 10: 422.

16. McCarthy DJ, Chen Y, Smyth GK. Differential expression analysis of multifactor RNA-Seq experiments with respect to biological variation. Nucleic Acids Res. 2012; 40: 4288-97.

17. Robinson MD, McCarthy DJ, Smyth GK. edgeR: a Bioconductor package for differential expression analysis of digital gene expression data. Bioinformatics. 2010; 26: 139-40.

18. Zhou Y, Zhou B, Pache L, Chang M, Khodabakhshi AH, Tanaseichuk O, et al. Metascape provides a biologist-oriented resource for the analysis of systems-level datasets. Nat Commun. 2019; 10: 1523.

19. Huang da W, Sherman BT, Lempicki RA. Systematic and integrative analysis of large gene lists using DAVID bioinformatics resources. Nat Protoc. 2009; 4: 44-57.

20. Szklarczyk D, Franceschini A, Wyder S, Forslund K, Heller D, Huerta-Cepas J, et al. STRING v10: protein-protein interaction networks, integrated over the tree of life. Nucleic Acids Res. 2015; 43: D447-52.

21. Shannon P, Markiel A, Ozier O, Baliga NS, Wang JT, Ramage D, et al. Cytoscape: a software environment for integrated models of biomolecular interaction networks. Genome Res. 2003; 13: 2498-504

22. Chin $\mathrm{CH}$, Chen $\mathrm{SH}, \mathrm{Wu} \mathrm{HH}, \mathrm{Ho} \mathrm{CW}, \mathrm{Ko} \mathrm{MT}$, Lin CY. cytoHubba: identifying hub objects and sub-networks from complex interactome. BMC Syst Biol. 2014; 8 Suppl 4: S11.

23. Bader GD, Hogue CW. An automated method for finding molecular complexes in large protein interaction networks. BMC Bioinformatics. 2003; 4: 2

24. Agarwal V, Bell GW, Nam JW, Bartel DP. Predicting effective microRNA target sites in mammalian mRNAs. Elife. 2015; 4

25. Wong $N$, Wang $X$. miRDB: an online resource for microRNA target prediction and functional annotations. Nucleic Acids Res. 2015; 43: D146-52.

26. Backes C, Kehl T, Stockel D, Fehlmann T, Schneider L, Meese E, et al. miRPathDB: a new dictionary on microRNAs and target pathways. Nucleic Acids Res. 2017; 45: D90-D6.

27. Dweep H, Gretz N. miRWalk2.0: a comprehensive atlas of microRNA-target interactions. Nat Methods. 2015; 12: 697.

28. Chen L, Jin T, Zhu K, Piao Y, Quan T, Quan C, et al. PI3K/mTOR dual inhibitor BEZ235 and histone deacetylase inhibitor Trichostatin A synergistically exert anti-tumor activity in breast cancer. Oncotarget. 2017; 8: 11937-49.

29. Zhang J, Wang L, Mao S, Liu M, Zhang W, Zhang Z, et al. miR-1-3p Contributes to Cell Proliferation and Invasion by Targeting Glutaminase in Bladder Cancer Cells. Cell Physiol Biochem. 2018; 51: 513-27.

30. Wang Y, Luo X, Liu Y, Han G, Sun D. Long noncoding RNA RMRP promotes proliferation and invasion via targeting miR-1-3p in non-small-cell lung cancer. J Cell Biochem. 2019; 120: 15170-81.

31. Zhang $\mathrm{H}$, Zhang $\mathrm{Z}$, Gao L, Qiao Z, Yu M, Yu B, et al. miR-1-3p suppresses proliferation of hepatocellular carcinoma through targeting SOX9. Onco Targets Ther. 2019; 12: 2149-57.

32. Zhi Y, Xie X, Wang R, Wang B, Gu W, Ling Y, et al. Serum level of miR-10-5p as a prognostic biomarker for acute myeloid leukemia. Int J Hematol. 2015; 102: 296-303

33. Tang Y, Pan I, Huang S, Peng X, Zou X, Luo Y, et al. Downregulation of miR-133a-3p promotes prostate cancer bone metastasis via activating PI3K/AKT signaling. J Exp Clin Cancer Res. 2018; 37: 160
34. Zhou Y, Jin Z, Wang C. Glycogen phosphorylase B promotes ovarian cancer progression via Wnt/beta-catenin signaling and is regulated by miR-133a-3p. Biomed Pharmacother. 2019; 120: 109449.

35. Shi W, Tang T, Li X, Deng S, Li R, Wang Y, et al. Methylation-mediated silencing of miR-133a-3p promotes breast cancer cell migration and stemness via miR-133a-3p/MAML1/DNMT3A positive feedback loop. J Exp Clin Cancer Res. 2019; 38: 429

36. Wang Z, Zhao Z, Yang $Y$, Luo $M$, Zhang M, Wang $X$, et al. MiR-99b-5p and miR-203a-3p Function as Tumor Suppressors by Targeting IGF-1R in Gastric Cancer. Sci Rep. 2018; 8: 10119.

37. Zhao YJ, Song X, Niu L, Tang Y, Song X, Xie L. Circulating Exosomal miR-150-5p and miR-99b-5p as Diagnostic Biomarkers for Colorectal Cancer. Front Oncol. 2019; 9: 1129.

38. Lukamowicz-Rajska M, Mittmann C, Prummer M, Zhong O, Bedke J, Hennenlotter J, et al. MiR-99b-5p expression and response to tyrosine kinase inhibitor treatment in clear cell renal cell carcinoma patients. Oncotarget. 2016; 7: 78433-47.

39. Tao S, Wang C, Chen Y, Deng Y, Song L, Shi Y, et al. Prognosis and outcome of patients with acute myeloid leukemia based on FLT3-ITD mutation with or without additional abnormal cytogenetics. Oncol Lett. 2019; 18: 6766-74.

40. Azari-Yam A, Tavakkoly-Bazzaz J, Semnani Y, Davoudi-Dehaghani E, Ghodssi-Ghassemabadi R, Kianfar S, et al. FLT3 Gene Mutation Profile and Prognosis in Adult Acute Myeloid Leukemia. Clin Lab. 2016; 62: 2011-7.

41. Bertacchini J, Heidari N, Mediani L, Capitani S, Shahjahani M, Ahmadzadeh A, et al. Targeting PI3K/AKT/mTOR network for treatment of leukemia. Cell Mol Life Sci. 2015; 72: 2337-47.

42. Martelli AM, Nyakern M, Tabellini G, Bortul R, Tazzari PL, Evangelisti C, et al. Phosphoinositide 3-kinase/Akt sionaling pathway and its therapeutical implications for human acute myeloid leukemia. Leukemia. 2006; 20: 911-28.

43. Kim SH, Turnbull J, Guimond S. Extracellular matrix and cell signalling: the dynamic cooperation of integrin, proteoglycan and growth factor receptor. J Endocrinol. 2011; 209: 139-51.

44. Li Y, Shi X, Yang W, Lu Z, Wang P, Chen Z, et al. Transcriptome profiling of lncRNA and co-expression networks in esophageal squamous cell carcinoma by RNA sequencing. Tumour Biol. 2016; 37: 13091-100.

45. Li S, Li H, Xu Y, Lv X. Identification of candidate biomarkers for epithelial ovarian cancer metastasis using microarray data. Oncol Lett. 2017; 14: 3967-74.

46. Eke I, Cordes N. Focal adhesion signaling and therapy resistance in cancer. Semin Cancer Biol. 2015; 31: 65-75.

47. Stewart G. The emerging physiological roles of the SLC14A family of urea transporters. Br J Pharmacol. 2011; 164: 1780-92.

48. Hou R, Kong X, Yang B, Xie Y, Chen G. SLC14A1: a novel target for human urothelial cancer. Clin Transl Oncol. 2017; 19: 1438-46.

49. Rafnar T, Vermeulen SH, Sulem P, Thorleifsson G, Aben KK, Witjes JA, et al. European genome-wide association study identifies SLC14A1 as a new urinary bladder cancer susceptibility gene. Hum Mol Genet. 2011; 20: 4268-81.

50. Li C, Jia L, Yu Y, Jin L. Lactic acid induced microRNA-744 enhances motility of $\mathrm{SiHa}$ cervical cancer cells through targeting ARHGAP5. Chem Biol Interact. 2019; 298: 86-95.

51. Dong G, Wang B, An Y, Li J, Wang X, Jia J, et al. SIRT1 suppresses the migration and invasion of gastric cancer by regulating ARHGAP5 expression. Cell Death Dis. 2018: 9: 977.

52. Fang Y, Zhu X, Wang J, Li N, Li D, Sakib N, et al. MiR-744 functions as a proto-oncogene in nasopharyngeal carcinoma progression and metastasis via transcriptional control of ARHGAP5. Oncotarget. 2015; 6: 13164-75.

53. Khwaja A. PI3K as a target for therapy in haematological malignancies. Curr Top Microbiol Immunol. 2010; 347: 169-88.

54. Konoplev S, Yin CC, Kornblau SM, Kantarjian HM, Konopleva M, Andreeff $\mathrm{M}$, et al. Molecular characterization of de novo Philadelphia chromosome-positive acute myeloid leukemia. Leuk Lymphoma. 2013; 54: 138-44.

55. Baohua $Y$, Xiaoyan Z, Tiecheng Z, Tao Q, Daren S. Mutations of the PIK3CA gene in diffuse large B cell lymphoma. Diagn Mol Pathol. 2008; 17: 159-65. 